\title{
Survivin expression modulates the sensitivity of A549 lung cancer cells resistance to vincristine
}

\author{
CHENGWEI ZHOU, YONGGANG ZHU, BIN LU, WEIJUN ZHAO and XIAODONG ZHAO
}

Thoracic Department, Affiliated Hospital of School of Medicine, Ningbo University, Ningbo, Zhejiang 315020, P.R. China

Received July 24, 2016; Accepted December 12, 2017

DOI: $10.3892 / \mathrm{ol} .2018 .9277$

\begin{abstract}
Lung cancer is the leading cause of cancer-associated mortalities worldwide. Chemotherapeutic drug vincristine is widely used to treat lung cancer; however, the acquisition of drug resistance is the major limitation of chemotherapy, and it is thus important to determine the mechanism underlying vincristine resistance in lung cancer. Survivin has been reported to be associated with the development of drug resistance and be involved in the progression of non-small cell lung cancer. In the present study, a vincristine-resistant lung cancer cell line, A549/VCR, was used to investigate the possible involvement of survivin in the acquisition of vincristine resistance. Western blot analysis demonstrated that survivin protein expression level was markedly higher in A549/VCR cells compared with in control A549 cells, whereas p53 expression level was lower in A549/VCR cells compared with in A549 cells. Thus, wild-type p53 was overexpressed in A549/VCR cells and it reversed vincristine resistance of A549/VCR cells via the inhibition of survivin expression. Furthermore, survivin was knocked down by small interfering RNA technology and the effects on viability and apoptosis of resistant cells were investigated. MTT, Annexin V-fluorescein isothiocyanate/propidium iodide and caspase-3 activity assays indicated that survivin silencing significantly inhibited cell viability and enhanced apoptosis induced by vincristine treatment in A549/VCR cells compared with non-silenced A549/VCR cells. These results suggested that survivin expression regulated by p53 may serve an important role in drug resistance in A549/VCR cells and may be a potential target for enhancing vincristine sensitivity in A549 lung cancer cells. Additionally, the present study revealed that A549/VCR cells exhibited cross resistance to methotrexate (MTX) and survivin silencing re-sensitized A549/VCR cells to MTX, indicating the crucial role of survivin in regulating A549 cells sensitivity to anticancer drugs. The results of the
\end{abstract}

Correspondence to: Dr Xiaodong Zhao, Thoracic Department, Affiliated Hospital of School of Medicine, Ningbo University, 247 Renmin Road, Ningbo, Zhejiang 315020, P.R. China

E-mail: zhxdong77@163.com

Key words: survivin, p53, drug resistance, vincristine, viability, apoptosis present study are significant for determining the underlying mechanism of vincristine resistance in lung cancer.

\section{Introduction}

Lung cancer is one of the most common types of malignant tumor, exhibiting the highest mortality rate worldwide due to the uncontrolled cell growth in tissues of the lung and high metastatic ability (1). Non-small cell lung cancer (NSCLC) accounts for $80-85 \%$ of lung cancer cases globally in 2015 (2). Anticancer drugs are widely used to treat lung cancer, and are frequently combined with surgical therapy and/or radiation therapy. However, the majority of patients are diagnosed at the advanced stage and NSCLC is highly resistant to the existing therapeutics, leading to poor prognosis $(3,4)$. Vincristine is a medication used to treat numerous types of cancer, including Hodgkin's disease, neuroblastoma, acute lymphocytic leukemia, acute myeloid leukemia, breast cancer and lung cancer. Vincristine serves its antitumor activity by inducing microtubule perturbation and inhibiting mitosis, thus resulting in cell apoptosis (5). However, the therapeutic efficacy was often unsatisfactory due to the development of drug resistance in lung cancer cells. Therefore, investigating the mechanism underlying vincristine resistance and exploring an effective therapy strategy to enhance the sensitivity of tumor cells to the drug are required to improve the efficacy of treatments for lung cancer.

It is widely considered that abnormal apoptosis inhibition during chemotherapy is involved in the development of drug resistance in tumor cells (6). Survivin is a member of the inhibitor of apoptosis protein family, encoded by the baculoviral inhibitor of apoptosis repeat-containing 5 gene in humans. It functions by accelerating cell proliferation and inhibiting apoptosis or programmed cell death (7). Survivin was revealed to be highly expressed in fetal tissues and the majority of human cancer types; however, it is rarely present in normal, non-malignant adult cells (8). It was also revealed that survivin demonstrated a higher expression level in lymph node metastases and tumor node metastasis stages, and a higher survivin expression level was revealed in NSCLC tissues compared with in normal tissues $(9,10)$. Additionally, survivin expression level was demonstrated to be associated with clinicopathological characteristics of NSCLC and may serve as a critical biomarker for NSCLC progression $(6,10)$. It was also revealed that survivin family proteins function as novel 
molecular determinants of doxorubicin resistance in organotypic human breast tumor (11). Additionally, survivin was reported to be serve a role in vincristine resistance in multiple myeloma cells (12). The molecular mechanisms underlying survivin regulation are still not well understood, but regulation of survivin has been suggested to be associated with p53 protein (13). It was reported that a high expression level of wild-type p53 repressed survivin expression at the mRNA level in ovarian cancer 2774qwl cells and lung cancer cells, and wild-type p53 expression induced by DNA-damaging agent adriamycin revealed a marked reduction in survivin expression level compared with in non-induced cells (14). The p53-wild type colorectal cancer cells exhibited greater cytotoxicity, apoptosis and survivin reduction compared with in the p53-null cancer cells following treatment with antitumor agent honokiol (15). Survivin and p53 expression have been observed to modulate honokiol-induced apoptosis in human colorectal cancer cells (15). Furthermore, p53 and survivin were demonstrated to serve an important role in cancer cell resistance to camptothecin-like chemotherapeutic agents (16).

The present study investigated the mechanism underlying vincristine resistance in A549 lung cancer cells, and indicated that the survivin level regulated by $\mathrm{p} 53$ expression was associated with resistance of A549 cells to vincristine, suggesting a potential candidate of therapeutic target for enhancing vincristine sensitivity in lung cancer.

\section{Materials and methods}

Cell culture. The A549 human NSCLC cell line was purchased from the Type Culture Collection of the Chinese Academy of Sciences (Shanghai, China). Cells were cultured in RPMI-1640 (Gibco; Thermo Fisher Scientific, Inc., Waltham, MA, USA) supplemented with $10 \%$ fetal bovine serum (FBS; Lonza Group, Ltd., Basel, Switzerland), 5 mM L-glutamine, $5 \mathrm{mM}$ non-essential amino acids, $100 \mathrm{U} / \mathrm{ml}$ penicillin and streptomycin (Invitrogen; Thermo Fisher Scientific, Inc.), in a humidified $5 \% \mathrm{CO}_{2}$ incubator at $37^{\circ} \mathrm{C}$.

Cultivation of vincristine-resistant cell line. Vincristineresistant A549 cells (A549/VCR) were developed by exposing A549 cells to an increasing concentration of vincristine ranging between 1 and $100 \mathrm{nM}$ in RPMI-1640 medium, as previously described (17). Briefly, A549 cells were cultured in $60 \mathrm{~mm}$ culture plates for $24 \mathrm{~h}$ and $1 \mathrm{nM}$ vincristine was added to the medium at $37^{\circ} \mathrm{C}$ for another $48 \mathrm{~h}$. Then medium was replaced with drug-free fresh medium to incubate the cells at $37^{\circ} \mathrm{C}$ for $48 \mathrm{~h}$ until confluency was reached. Subsequently, the cells were trypsizined, replated and were re-exposed to double the dose of drug. This process was repeated until the cells demonstrated resistance to $100 \mathrm{nM}$ vincristine. Following exposure to increasing concentrations of vincristine for at least 3 months, living cells were collected and classified as drug resistant cells (A549/VCR), which were used for subsequent experiments.

Construction of wild-type p53-overexpressed cell line. A recombinant replication-defective adenoviral vector containing wild-type p53 (rAdV-p53; 1x1012 vp/ml) and a control vector were obtained from Sibiono GeneTech Co., Ltd. (Shenzhen,
China). A total of $4 \times 10^{4}$ A549/VCR cells were seeded in a 24-well plate for $\mathrm{rAdV}$ infection. Cells were infected with rAdV-p53 $\left(1 \times 10^{7}\right.$ copies $\left./ \mathrm{ml}\right)$ using Lipofectamine ${ }^{\mathrm{TM}} 2000$ (Invitrogen; Thermo Fisher Scientific, Inc.) according to the manufacturer's protocol, and then inoculated in a 96-well plate and treated with $100 \mathrm{nM}$ vincristine for $48 \mathrm{~h}$ at $37^{\circ} \mathrm{C}$. These cells were prepared for viability analysis.

Cell viability assay. Cell viability was evaluated using a MTT assay (Sigma-Aldrich; Merck KGaA, Darmstadt, Germany). A total of 1x104 cells were seeded into each well of a 96-well plate in $100 \mu$ l RPMI-1640 supplemented with 10\% FBS at $37^{\circ} \mathrm{C}$ with $5 \% \mathrm{CO}_{2}$ for $24 \mathrm{~h}$. The cells were then incubated with various concentrations of vincristine $(0,10,50,150,300,600$ and $1,200 \mathrm{nM})$ or $(0,0.01,0.1,1,10,100$ and $1,000 \mu \mathrm{M})$ methotrexate (MTX, cat. no. C13876, Chemleader, Shanghai, China) for $48 \mathrm{~h}$, respectively. Subsequently, $20 \mu \mathrm{l}$ MTT $(5 \mathrm{mg} / \mathrm{ml}$ in PBS) was added to each well and incubated for $4 \mathrm{~h}$ at $37^{\circ} \mathrm{C}$, and then cells were lysed for $10 \mathrm{~min}$ by addition of $200 \mu \mathrm{l}$ dimethylsulfoxide (OriGene Technologies, Inc., Rockville, MD, USA) at room temperature. Absorbance was evaluated at $570 \mathrm{~nm}$ using a Rainbow microplate reader (Tecan Group, Ltd., Mannedorf, Switzerland). Cell viability was expressed as a ratio relative to the absorbance obtained from the well containing non-treated control cells.

Western blot analysis. Cells were seeded in a culture dish and cultured overnight, prior to incubation with 0,50 or $100 \mathrm{nM}$ vincristine for $48 \mathrm{~h}$. Cells were harvested, washed twice using cold PBS and lysed in Western and IP cell lysate buffer (cat. no. P0013; Beyotime Institute of Biotechnology, Haimen, China), containing a protease inhibitor cocktail (Roche Diagnostics, Basel, Switzerland) at $4^{\circ} \mathrm{C}$ for $15 \mathrm{~min}$. Following centrifugation at $12,000 \mathrm{x}$ g for $10 \mathrm{~min}$ at $4^{\circ} \mathrm{C}$, the supernatant was collected and quantified using a bicinchoninic acid quantification kit (Beyotime Institute of Biotechnology). The protein $(50 \mu \mathrm{g})$ was separated by $10 \%$ SDS-PAGE (Beijing Solarbio Science \& Technology Co., Ltd., Beijing, China) and transferred to polyvinylidene fluoride membranes (EMD Millipore, Billerica, MA, USA). The membranes were blocked with 5\% non-fat dried milk in TBS Tween-20 for $1 \mathrm{~h}$ at room temperature and incubated overnight at $4^{\circ} \mathrm{C}$ with specific primary antibodies: Rabbit polyclonal anti-survivin (1:500; cat. no. sc-10811), mouse monoclonal anti-p53 (1:1,000; cat. no. sc-126), rabbit polyclonal anti-GAPDH (1:2,000; cat. no. sc-25778), all purchased from Santa Cruz Biotechnology, Inc. (Dallas, TX, USA). Horseradish peroxidase-conjugated secondary antibodies: Goat anti-mouse $(1: 2,000$; cat. no. sc-2005) and goat anti-rabbit IgG (1:2,000; cat. no. sc-2004) (all from Santa Cruz Biotechnology, Inc.) were used for incubation at room temperature for $2 \mathrm{~h}$. Development was performed using enhanced chemiluminescence-detecting reagent (GE Healthcare, Chicago, IL, USA). The protein blots were quantified by densitometry using QuantityOne version 4.6.2 software (Bio-Rad Laboratories, Inc., Hercules, CA, USA) and the amounts were expressed relative to the internal reference, GAPDH.

RNA interference of survivin. Short interfering (si)RNAs against survivin and the negative control were designed, 
A

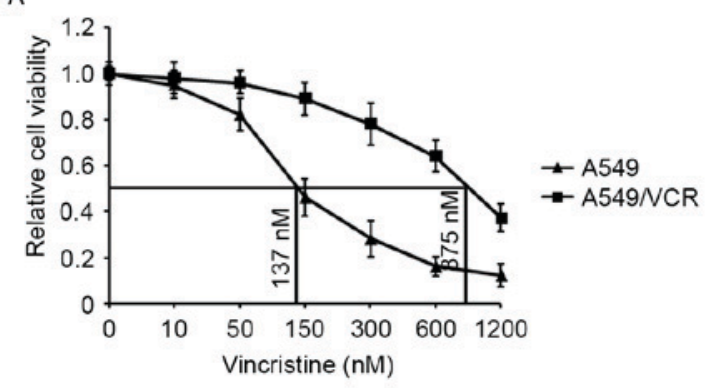

B

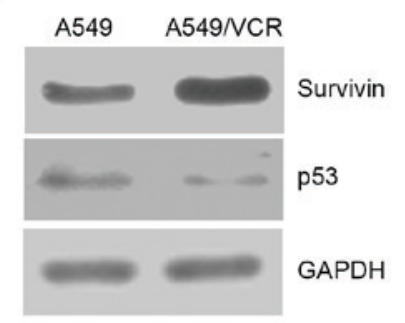

c

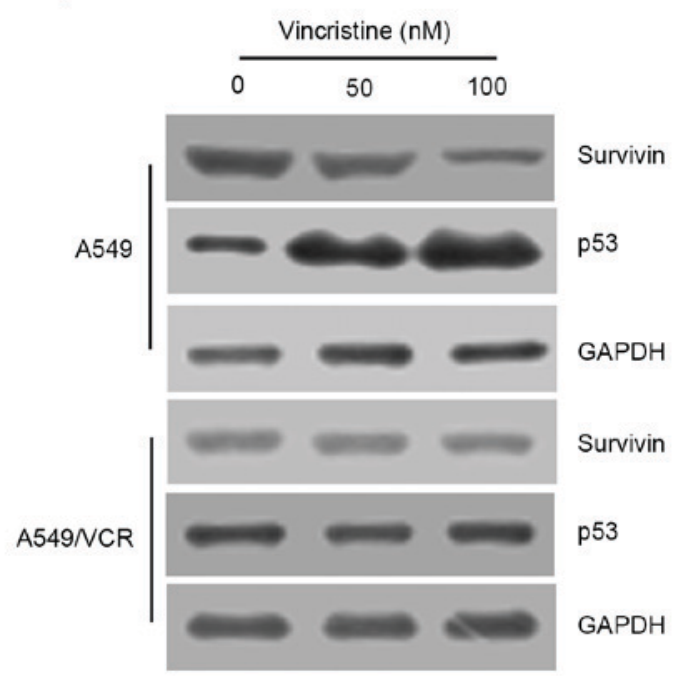

Figure 1. Characterization of A549/VCR and A549 cells. (A) The effect of vincristine on viability in A549/VCR and A549 cells was evaluated by MTT assay. A growth curve was plotted and half-maximal inhibitory concentration values of vincristine in the two types of cells were determined, respectively. (B) Survivin and p53 expression levels in A549/VCR and A549 cells were analyzed by western blot analysis using anti-survivin or anti-p53 antibodies. (C) The effect of vincristine on survivin and p53 expression levels in A549/VCR and A549 cells were evaluated by western blot analysis. GAPDH was used as an internal standard. VCR, vincristine-resistant.

and chemically synthesized by GenePham, Inc. (Sunnyvale, CA, USA). Cell transfections were performed using Lipofectamine ${ }^{\circledR} 2000$ (Invitrogen; Thermo Fisher Scientific, Inc.), according to the manufacturer's protocol. Cells were cultured for $48 \mathrm{~h}$ following transfection, and subsequently the cells were lysed and protein expression levels of survivin were analyzed by western blotting as aforementioned.

Apoptosis assay. Transfected cells were treated with or without $100 \mathrm{nM}$ vincristine for $48 \mathrm{~h}$. Apoptosis was analyzed using Annexin V-fluorescein isothiocyanate (FITC)/propidium iodide (PI) assay (BD Biosciences, San Jose, CA, USA), according to the manufacturer's protocol. The amount of phosphatidylserine on the outer surface of the plasma membrane (a biochemical alteration unique to membranes of apoptotic cells) and the amount of PI, a dye that easily enters dead cells or cells in the late stages of apoptosis and binds DNA, but does not bind the plasma membrane of viable cells, were detected. Fluorescence was detected using a FACSCalibur flow cytometer (BD Biosciences) by fluorescence activated cell sorter analysis, and data were analyzed using CellQuest software (BD Biosciences). Cells with phosphatidylserine on their surface were considered to be apoptotic. To verify the effect on apoptosis, caspase-3 activation was also determined using the human active caspase-3 ELISA kit (cat. no. ABIN1568799; R\&D Systems, Inc., Minneapolis, MN, USA). Survivin siRNA-transfected or control siRNA-transfected cells were treated with or without $100 \mathrm{nM}$ vincristine. Following a 24-h incubation, the cells were lysed and assayed as described by the manufacturer.

Statistical analysis. Data are expressed as the mean \pm standard error of the mean. Statistical analyses were performed using SPSS software (version 11.0; SPSS, Inc., Chicago, IL, USA).
All of the experiments were repeated at least three times. One-way analysis of variance (ANOVA) was used to assess differences between groups. Duncan's new multiple range test as a post hoc test following ANOVA was employed for pairwise comparison followed by Bonferroni correction. $\mathrm{P}<0.05$ was considered to indicate a statistically significant difference.

\section{Results}

Characterization of vincristine-resistant cells. To investigate the molecular mechanism underlying acquired drug resistance in A459 lung cancer cells, the A549/VCR cell model system was established. A549/VCR and A549 cells were exposed to vincristine at the indicated concentrations $(0,10,50,150$, 300, 600 and 1,200 $\mathrm{nM}$ ) for $48 \mathrm{~h}$. Cell viability was detected using the MTT assay and the growth curve was analyzed. The half-maximal inhibitory concentration $\left(\mathrm{IC}_{50}\right)$ of vincristine in A549/VCR cells was determined to be $875 \mathrm{nM}$ and that of A549 cells was $137 \mathrm{nM}$ at $48 \mathrm{~h}$ (Fig. 1A). Subsequently, survivin expression level in A549/VCR and A549 cells was evaluated by western blot analysis. The results demonstrated that the expression level of survivin in A549/VCR cells was markedly higher compared with in A549 cells, whereas the expression level of p53 was lower in A549/VCR cells compared with in A549 cells (Fig. 1B). Additionally, A549/VCR and A549 cells were treated with 0,50 or $100 \mathrm{nM}$ vincristine for 48 h. p53 expression level was revealed to be increased by vincristine treatment in a concentration-dependent manner in A549 cells, whereas it remained relatively constant at low levels in A549/VCR cells following vincristine treatment (Fig. 1C). Survivin expression level was decreased by vincristine in a concentration-dependent manner in A549 cells, but not in A549/VCR cells. These results indicated that the 
vincristine resistant lung cancer A549 cells were successfully constructed, and survivin and p53 may serve a critical role in mediating vincristine resistance in A549 cells.

p53 overexpression re-sensitizes A549/VCR cells to vincristine via survivin inhibition. To confirm whether p53 and survivin are associated with vincristine resistance in A549 lung cancer cells, wild-type p53 was overexpressed in A549/VCR cells by $\mathrm{rAdV}-\mathrm{p} 53$ infection. Following infection for $24 \mathrm{~h}$, cells were treated with $100 \mathrm{nM}$ vincristine for $48 \mathrm{~h}$. Subsequently, cell viability was assessed by MTT assay and the expression level of survivin was determined by western blotting. The results revealed that p53 overexpression in A549/VCR cells markedly enhanced the inhibition of cell viability induced by vincristine, with decreased survivin expression, compared with the vincristine-treated control A549/VCR cells (Fig. 2). The results suggested that $\mathrm{p} 53$ overexpression may re-sensitize A549/VCR cells to vincristine by inhibiting survivin expression, and vincristine resistance may have be induced by the increase of survivin expression mediated by p53 expression level reduction.

Survivin silencing enhances the sensitivity of A549/VCR cells to vincristine. In order to investigate the role of survivin in vincristine-resistance A549 cells lung cancer cells, survivin expression was knocked down using siRNA technology. Western blot analysis demonstrated that the protein expression level of survivin was markedly inhibited in survivin siRNA transfected A549/VCR and A549 cells compared with the control siRNA transfected cells (Fig. 3A). Survivin-silenced or non-silenced cells were treated with or without $100 \mathrm{nM}$ vincristine for $48 \mathrm{~h}$. The cell viability assay demonstrated that survivin silencing significantly inhibited the viability of A549/VCR cells treated with vincristine compared with non-silenced resistant cells treated with vincristine alone (Fig. 3B). Furthermore, survivin silencing also increased the inhibition of vincristine treatment on A549 cell viability compared with non-silenced A549 cells (Fig. 3B). These results revealed that survivin silencing enhanced the sensitivity of A549/VCR and A549 cells to vincristine.

Survivin silencing accelerates apoptosis of A549/VCR cells induced by vincristine. In order to clarify the cause of the re-sensitization of A549/VCR cells mediated by survivin silencing to vincristine, cell apoptosis was analyzed using an Annexin V-FITC/PI assay. Survivin-silenced or non-silenced A549/VCR, and A549 cells were respectively treated with or without $100 \mathrm{nM}$ vincristine for $48 \mathrm{~h}$. The apoptosis assay indicated that apoptosis in survivin-silenced A549/VCR resistant cells was significantly increased by vincristine treatment compared with the non-silenced resistant cells upon vincristine treatment alone (Fig. 4A). Additionally, under identical experimental conditions, apoptosis of A549 cells was also increased. Furthermore, caspase-3 activity was evaluated using an ELISA assay to verify the effect of survivin silencing on apoptosis in resistant cells induced by vincristine. The results of the ELISA assay supported the aforementioned observations on the apoptosis rate, whereby surviving silencing significantly increased active caspase-3 activity following vincristine treatment (Fig. 4B). These results suggested that survivin
A

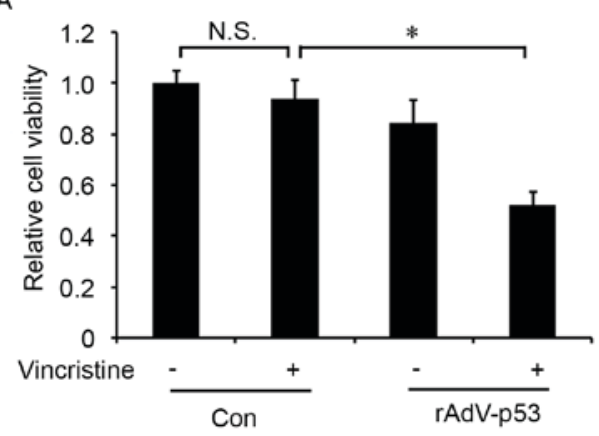

B

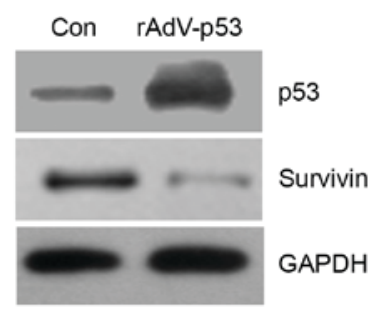

Figure 2. Effect of p53 overexpression on vincristine sensitivity in A549/VCR cells. (A) Wild-type p53 was overexpressed in A549/VCR cells by rAdV-p53 infection and cells were treated with or without $100 \mathrm{nM}$ vincristine. Cell viability was analyzed by MTT assay. ${ }^{*} \mathrm{P}<0.05$. (B) Protein expression levels of survivin and p53 in p53-overexpressed (rAdV-p53) and control rAdV-infected A549/VCR cells (Con) were examined by western blot analysis using the respective antibodies. GAPDH was detected as an internal standard. N.S.; no significance; Con, control; VCR, vincristine-resistant.

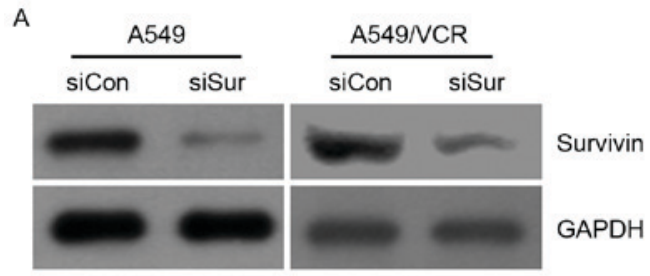

B

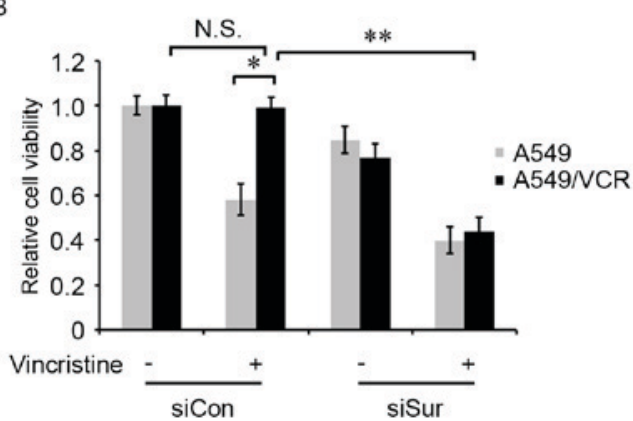

Figure 3. Survivin silencing re-sensitizes A549/VCR cells to vincristine. A549/VCR and A549 cells were transfected with siCon or siSur for $48 \mathrm{~h}$. (A) Protein expression levels of survivin in A549/VCR and A549 cells were analyzed by western blotting. (B) Survivin-silenced or non-silenced A549/VCR and A549 cells were treated with or without $100 \mathrm{nM}$ vincristine. Cell viability was detected by MTT assay. ${ }^{*} \mathrm{P}<0.05 ;{ }^{* * *} \mathrm{P}<0.01 ;$ N.S., no significance; siCon, control short interfering RNA; siSur, survivin short interfering RNA; VCR, vincristine-resistant.

may be associated with vincristine resistance of lung cancer A549 cells and survivin silencing may increase the sensitivity of resistant cells to vincristine by enhancing the apoptosis induced by vincristine. 


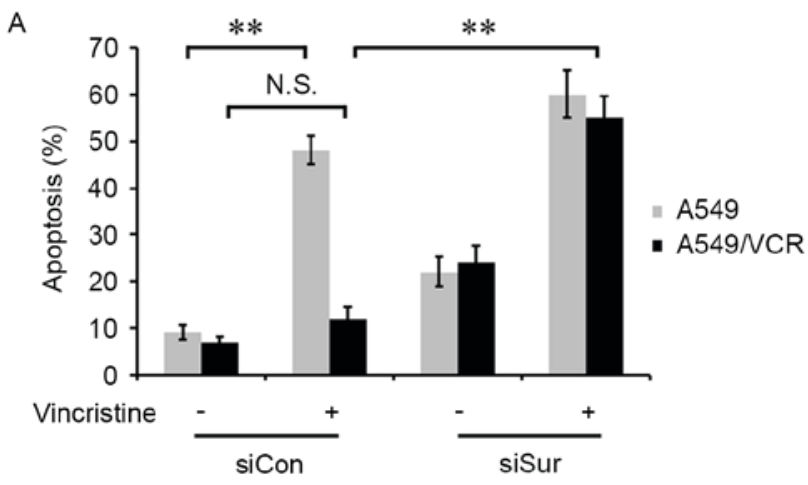

B

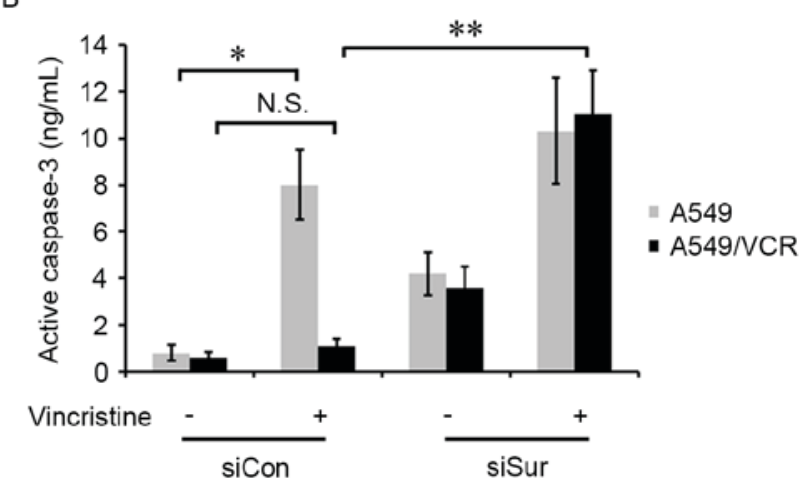

Figure 4. Effect of survivin silencing on the apoptosis induced by vincristine in A549/VCR cells. A549/VCR and A549 cells were transfected with siCon and siSur for $24 \mathrm{~h}$, and were then exposed to $100 \mathrm{nM}$ vincristine. (A) Apoptosis of A549/VCR and A549 cells were respectively evaluated by Annexin V-fluorescein isothiocyanate/propidium iodide assay. (B) Caspase-3 activity in Survivin-silenced or non-silenced A549/VCR and A549 cells treated with or without vincristine was evaluated by ELISA assay. ${ }^{*} \mathrm{P}<0.05$; ${ }^{* *} \mathrm{P}<0.01$. N.S., no significance; siCon, control short interfering RNA; siSur, survivin short interfering RNA; VCR, vincristine-resistant.

Survivin silencing re-sensitizes A549/VCR cells to anticancer drug MTX. The present study subsequently investigated the drug resistance of A549/VCR cells to other anticancer drugs. A549 and A549/VCR cells were plated and treated with various concentrations of anticancer drug MTX. Following drug treatment for $48 \mathrm{~h}$, cell viability was evaluated by MTT assay. The results revealed that A549/VCR cells exhibited cross resistance to MTX (Fig. 5A). Survivin-silenced or non-silenced A549/VCR and A549 cells were treated with, or without $1 \mu \mathrm{M}$ MTX for $48 \mathrm{~h}$. Cell viability was detected using an MTT assay. The present study demonstrated that survivin silencing significantly enhanced resistant A549/VCR cells sensitivity to MTX (Fig. 5B). These results suggested that survivin may serve an important role in improving the drug sensitivity of resistant A549 cells.

\section{Discussion}

Lung cancer, as one of the most common types of malignant tumor worldwide, seriously threatens human health (1). Current standard treatment of lung cancer consists of complete cytoreductive surgery followed by chemotherapy. However, the development of drug resistance is the leading cause of chemotherapy failure. As vincristine is widely used in clinical treatment, the present study focused on further characterizing

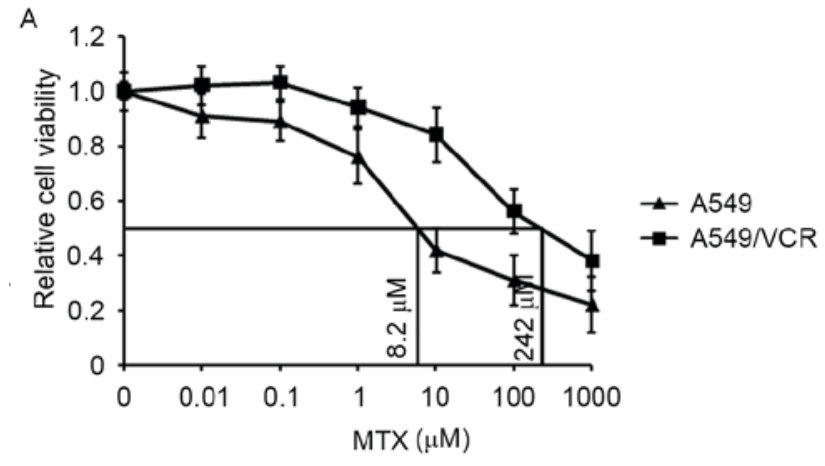

B

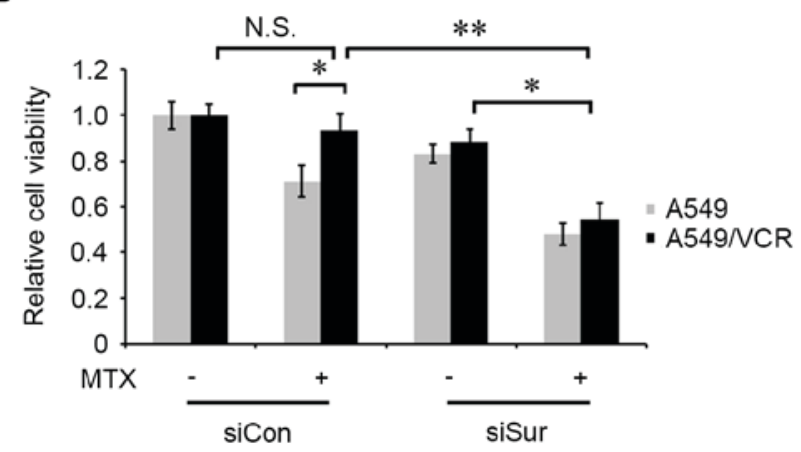

Figure 5. Survivin silencing enhances the sensitivity of A549/VCR cells to methotrexate. (A) A549/VCR exhibited cross-resistance to MTX. A549 and A549/VCR were plated and treated with various concentrations of MTX. Following drug treatment for $48 \mathrm{~h}$, the drug-containing medium was replaced and cell viability was evaluated by MTT assay. (B) Survivin-silenced or non-silenced A549/VCR and A549 cells were treated with or without $1 \mu \mathrm{M}$ MTX. Cell viability was detected by MTT assay. ${ }^{*} \mathrm{P}<0.05 ;{ }^{* *} \mathrm{P}<0.01 ; \mathrm{N} . \mathrm{S}$., no significance; MTX, methotrexate; VCR, vincristine-resistant.

vincristine-resistant cells and investigated the underlying mechanism.

The present study developed A549/VCR vincristine-resistant lung cancer cells by simulating the condition of drug resistance development in vivo using increasing concentrations of vincristine. The cell viability assay demonstrated that the $\mathrm{IC}_{50}$ of vincristine in drug resistant A549/VCR cells at $48 \mathrm{~h}$ was significantly higher compared with that in parental A549 cells, and vincristine at a concentration of $100 \mathrm{nM}$ did not significantly affect the viability of A549/VCR cells compared with A549 cells, suggesting that A549/VCR cells revealed resistance to vincristine and may be used in further experiments.

Chemotherapy kills tumor cells by activating apoptosis, whereas apoptotic pathway defects are associated with tumor resistance to chemotherapy (18). Vincristine exerts its antitumor activity by inducing microtubule perturbation and inhibiting mitosis, thus resulting in cell apoptosis (5). Survivin is an inhibitor of apoptosis protein, promoting cell proliferation and inhibiting apoptosis (7). Survivin was demonstrated to be associated with the progression and development of tumors, and is often overexpressed in cancer tissues (8-10). Additionally, survivin was also reported to be involved in the development of drug resistance in cancer cells $(11,12)$. The present study revealed that the survivin expression level in A549/VCR cells was markedly higher compared with in A549 cells, suggesting its participation in vincristine resistance. The molecular mechanisms underlying survivin regulation are still not well understood. It was 
previously demonstrated that survivin is a direct target gene of the Wnt signaling pathway and is upregulated by $\beta$-catenin (19). It was also indicated that survivin is increased via activation of extracellular signal-regulated kinase $1 / 2$, protein kinase $B$ (Akt) and nuclear factor $(\mathrm{NF})-\kappa \mathrm{B}$ in vincristine resistance in numerous types of myeloma cells (12). The present study revealed that survivin expression levels were associated with p53 expression level in inducing vincristine resistance in lung cancer A549 cells. The normal function of p53 is to regulate genes that control apoptosis, and survivin is a known inhibitor of apoptosis, thus p53 repression of survivin is implied to be a mechanism by which cells can undergo apoptosis induced by apoptotic stimuli or signals. Conversely, when survivin was overexpressed in cancer cells, including A549/VCR cells, the apoptotic response was significantly reduced, thus exhibiting vincristine resistance, as mentioned in a previous study (14). Therefore, downregulation of survivin by wild-type p53 overexpression is important for vincristine-induced A549/VCR cells apoptosis, which re-sensitizes A549/VCR cells to vincristine. Additionally, it was reported that the effect of the inhibition of p53 on survivin was performed by binding a specific region proximal to the transcription start site of survivin and affecting the modification of the chromatin inside the promoter region, resulting in transcriptional repression of the survivin gene (14). Identification of the molecules involved in the regulation of survivin in vincristine resistance lung cancer A549 cells remains to be established.

p53 regulates a variety of cellular functions, including DNA repair, cell cycle arrest and apoptosis (20-22). Abnormal expression of p53 has been observed in nearly all types of cancer, and p53 mutations are associated with resistance to chemotherapeutics and poor patient prognosis (23-26). The present study demonstrated that p53-null A549 cells produced by via a p53-siRNA knockdown revealed a high level of survivin and were resistant to vincristine-mediated apoptosis induction following vincristine treatment for $48 \mathrm{~h}$ (data not shown). Conversely, A549 cells with wild-type p53 demonstrated a low-level expression of survivin and were sensitive to vincristine-mediated apoptosis. These results revealed that the sensitivity of lung cancer A549 cells to vincristine was dependent on p53 status, and that survivin was associated with vincristine resistance in A549 cells. p53 is activated in response to numerous stressors, including DNA damage induced by either ultraviolet, ionizing radiation or chemical agents, oxidative stress, osmotic shock, ribonucleotide depletion and deregulated oncogene expression (27). The half-life of the p53 protein is increased following stress for rapid accumulation or a conformational change occurs as a transcription regulator by the phosphorylation of its $\mathrm{N}$-terminal domain (27). In the present study, p53 protein expression levels in A549 cells was increased by vincristine treatment for $48 \mathrm{~h}$, whereas acquired-resistant A549/VCR cells exhibited low p53 expression levels compared with in A549 cells. p53 was downregulated via a continuous proteasome degradation mediated by ubiquitin ligase, including mouse double minute 2 homolog and casein kinase $2(28,29)$. However, further investigation is required to decipher the mechanism underlying the reduction in p53 protein levels.

Survivin has been investigated as a critical biomarker for cancer progression and as a candidate target for therapy to enhance the sensitivity to chemotherapy by regulating proliferation or apoptosis-associated protein expression (30). The present study revealed that inhibition of survivin by siRNA enhanced the sensitivity of A549/VCR cells to vincristine by inducing cell apoptosis. It has been indicated that antisense treatment against survivin sensitized resistant HT29 human colon cancer cells to MTX (31). The present study demonstrated that A549/VCR cells exhibited cross resistance to MTX and survivin silencing re-sensitized A549/VCR cells to MTX, indicating the crucial role of survivin in regulating A549 cells sensitivity to anticancer drugs. It was previously reported that YM155, a novel small-molecule inhibitor of survivin, may decrease phosphorylated phosphoinositide-3 kinase and Akt expression levels in osteosarcoma cell lines, and suppress tumor growth (32). Tamm et al (33) revealed that survivin inhibited apoptosis regulator Bax and Fas-induced apoptotic signaling pathways in HEK293 cells. In addition, survivin blocked apoptosis by directly inhibiting caspases (caspase-3 or -7) to prevent a cascade of cleavage and activation amplification from occurring (32). It was demonstrated that survivin siRNA combined with the neoadjuvant chemotherapy significantly increased the sensitivity of MCF-7 breast cancer cells to chemotherapeutic agents paclitaxel and epirubicin, thus increasing the cell apoptosis rate (34). It was also revealed that survivin attenuates the suppressive effect of p53 on $\mathrm{NF}-\kappa \mathrm{B}$ (p50) expression and increases breast cancer resistance protein expression levels, thus modulate multidrug resistance in MCF-7 cells (35). However, the molecular mechanism underlying survivin regulating vincristine sensitivity in A549 cells remains unclear and requires further investigation.

In conclusion, survivin is a critical regulator in the development of vincristine resistance in A549 lung cancer cells. The results of the present study demonstrated that gene treatment targeting resistance-associated protein survivin combined with chemotherapy may be a more effective way for improving the sensitivity of lung cancer A549 cells to vincristine. To verify the universality of the mechanism in lung cancer and thus improve therapy efficiency, other lung cancer cell lines should be investigated.

\section{Acknowledgements}

Not applicable.

\section{Funding}

No funding was received.

\section{Availability of data and materials}

The datasets generated and/or analyzed during the current study are not publicly available due to use in future studies but are available from the corresponding author on reasonable request.

\section{Authors' contributions}

$\mathrm{CZ}$ and $\mathrm{XZ}$ were the major contributors in conception and design of the research and revision of manuscript for important intellectual content. Acquisition of data was performed by $\mathrm{YZ}$ and $\mathrm{BL}$. WZ was the major contributor in analysis and interpretation of data and statistical analysis. 


\section{Ethics approval and consent to participate}

Not applicable.

\section{Consent for publication}

Not applicable.

\section{Competing interests}

The authors declare that they have no competing interests.

\section{References}

1. National Cancer Institute (NCI): PDQ Cancer Information Summaries [Internet]. Non-Small Cell Lung Cancer Treatment $\left(\mathrm{PDQ}^{\circledR}\right)$, Patient Version. NCI, Bethesda, MD, 2002.

2. Zhang Y, Qin Q, Li B, Wang J and Zhang K: Magnetic resonance imaging for $\mathrm{N}$ staging in non-small cell lung cancer: A systematic review and meta-analysis. Thorac Cancer 6: 123-132, 2015.

3. Du Y, Su T, Zhao L, Tan X, Chang W, Zhang H and Cao G: Associations of polymorphisms in DNA repair genes and MDR1 gene with chemotherapy response and survival of non-small cell lung cancer. PLoS One 9: e99843, 2014.

4. Chen WL, Kuo KT, Chou TY, Chen CL, Wang CH, Wei YH and Wang LS: The role of cytochrome c oxidase subunit Va in non-small cell lung carcinoma cells: Association with migration, invasion and prediction of distant metastasis. BMC Cancer 12 273, 2012.

5. Jordan MA: Mechanism of action of antitumor drugs that interact with microtubules and tubulin. Curr Med Chem Anticancer Agents 2: 1-17, 2002.

6. Akyürek N, Memiş L, Ekinci $\mathrm{O}$, Köktürk $\mathrm{N}$ and Oztürk $\mathrm{C}$ : Survivin expression in pre-invasive lesions and non-small cell lung carcinoma. Virchows Arch 449: 164-170, 2006.

7. Jha K, Shukla M and Pandey M: Survivin expression and targeting in breast cancer. Surg Oncol 21: 125-131, 2012.

8. Sah NK, Khan Z, Khan GJ and Bisen PS: Structural, functional and therapeutic biology of survivin. Cancer Lett 244: 164-171, 2006.

9. Halasova E, Adamkov M, Matakova T, Vybohova D, Antosova M, Janickova M, Singliar A, Dobrota D and Jakus V: Expression of $\mathrm{Ki}-67, \mathrm{Bcl}-2$, survivin and p53 proteins in patients with pulmonary carcinoma. Adv Exp Med Biol 756: 15-21, 2013.

10. Duan L, Hu X, Jin Y, Liu R and You Q: Survivin protein expression is involved in the progression of non-small cell lungcancer in Asians: A meta-analysis. BMC Cancer 16: 276, 2016

11. Faversani A, Vaira V, Moro GP, Tosi D, Lopergolo A, Schultz DC, Rivadeneira D, Altieri DC and Bosari S: Survivin family proteins as novel molecular determinants of doxorubicin resistance in organotypic human breast tumors. Breast Cancer Res 16: R55, 2014.

12. Tsubaki M, Takeda T, Ogawa N, Sakamoto K, Shimaoka H, Fujita A, Itoh T, Imano M, Ishizaka T, Satou T and Nishida $S$ Overexpression of survivin via activation of ERK1/2, Akt, and $\mathrm{NF}-\kappa \mathrm{B}$ plays a central role invincristine resistance in multiple myeloma cells. Leuk Res 39: 445-452, 2015.

13. Xu Y, Wang S, Chan HF, Lu H, Lin Z, He C and Chen M: Dihydromyricetin induces apoptosis and reverses drug resistance in ovarian cancer cells by p53-mediated downregulation of survivin. Sci Rep 7: 46060, 2017.

14. Mirza A, McGuirk M, Hockenberry TN, Wu Q, Ashar H, Black S, Wen SF, Wang L, Kirschmeier P, Bishop WR, et al: Human survivin is negatively regulated by wild-type p53 and participates in p53-dependent apoptotic pathway. Oncogene 21: 2613-2622, 2002.

15. Lai YJ, Lin CI, Wang CL and Chao JI: Expression of survivin and p53 modulates honokiol-induced apoptosis in colorectal cancer cells. J Cell Biochem 115: 1888-1899, 2014.
16. Tomicic MT and Kaina B: Topoisomerase degradation, DSB repair, 53 and IAPs in cancer cell resistance to camptothecin-like topoisomerase I inhibitors. Biochim Biophys Acta 1835: 11-27, 2013.

17. Meena AS, Sharma A, Kumari R, Mohammad N, Singh SV and Bhat MK: Inherent and acquired resistance to paclitaxel in hepatocellular carcinoma: Molecular eventsinvolved. PLoS One 8: e61524, 2013.

18. Mohamed H, Hidemichi W, Ali AA, Yusuke O and Noriaki S: Apoptosis and molecular targeting therapy in cancer. Biomed Res Int 2014: 23, 2014.

19. Olie RA, Simões-Wüst AP, Baumann B, Leech SH, Fabbro D, Stahel RA and Zangemeister-Wittke U: A novel antisense oligonucleotide targeting survivin expression induces apoptosis and sensitizes lung cancer cells to chemotherapy. Cancer Res 60: 2805-2809, 2000

20. Riley T, Sontag E, Chen P and Levine A: Transcriptional control of human p53-regulated genes. Nat Rev Mol Cell Biol 9: 402-412, 2008.

21. García-Cao I, García-Cao M, Martín-Caballero J, Criado LM, Klatt P, Flores JM, Weill JC, Blasco MA and Serrano M: 'Super p53' mice exhibit enhanced DNA damage response, are tumor resistant and age normally. EMBO J 21: 6225-6235, 2002.

22. Vogelstein B, Lane D and Levine AJ: Surfing the p53 network. Nature 408: 307-310, 2000

23. Soussi T and Béroud C: Assessing TP53 status in human tumours to evaluate clinical outcome. Nat Rev Cancer 1: 233-240, 2001.

24. Papadogianni D, Soulitzis N, Delakas D and Spandidos DA: Expression of p53 family genes in urinary bladder cancer: Correlation with disease aggressiveness and recurrence. Tumour Biol 35: 2481-2489, 2014.

25. Shetzer Y, Solomon H, Koifman G, Molchadsky A, Horesh S and Rotter V: The paradigm of mutant $\mathrm{p} 53$-expressing cancer stem cells and drug resistance. Carcinogenesis 35: 1196-1208, 2014.

26. Kim CW, Lu JN, Go SI, Jung JH, Yi SM, Jeong JH, Hah YS, Han MS, Park JW, Lee WS and Min YJ: p53 restoration can overcome cisplatin resistance through inhibition of Akt as well as induction of Bax. Int J Oncol 43: 1495-1502, 2013.

27. Han ES, Muller FL, Pérez VI, Qi W, Liang H, Xi L, Fu C, Doyle E, Hickey M, Cornell J, et al: The in vivo gene expression signature of oxidative stress. Physiol Genomics 34: 112-126, 2014, 2008.

28. Hock AK and Vousden KH: The role of ubiquitin modification in the regulation of p53. Biochim Biophys Acta 1843: 137-149, 2014.

29. Hubert A, Paris S, Piret JP, Ninane N, Raes M and Michiels C: Casein kinase 2 inhibition decreases hypoxia-inducible factor-1 activity under hypoxia through elevated p53 protein level. J Cell Sci 119: 3351-3362, 2006.

30. Kelly RJ, Lopez-Chavez A, Citrin D, Janik JE and Morris JC: Impacting tumor cell-fate by targeting the inhibitor of apoptosis protein survivin. Mol Cancer 10: 35, 2011.

31. Peñuelas S, Noé V and Ciudad CJ: Modulation of IMPDH2, survivin, topoisomerase I and vimentin increases sensitivity to methotrexate in HT29 human colon cancer cells. FEBS J 272: 696-710, 2005

32. Zhang Z, Ma L and Wang J: YM155 exerts a growth inhibitory effect on human osteosarcoma in vitro and in vivo. Oncol Rep 34 1074-1080, 2015

33. Tamm I, Wang Y, Sausville E, Scudiero DA, Vigna N, Oltersdorf T and Reed JC: IAP-family protein survivin inhibits caspase activity and apoptosis induced by Fas (CD95), Bax, caspases, and anticancer drugs. Cancer Res 58: 5315-5320, 1998.

34. Dong H, Yao L, Bi W, Wang F, Song W and Lv Y: Combination of survivin siRNA with neoadjuvant chemotherapy enhances apoptosis and reversesdrug resistance in breast cancer MCF-7 cells. J Cancer Res Ther 11: 717-722, 2015.

35. Wang QP, Wang Y, Wang XD, Mo XM, Gu J, Lu ZY, Pan ZL and Zhu YX: Survivin up-regulates the expression of breast cancer resistance protein (BCRP) through attenuating the suppression of $\mathrm{p} 53$ on NF- $\mathrm{KB}$ expression in MCF-7/5-FU cells. Int J Biochem Cell Biol 45: 2036-2044, 2013. 